(C) The Author 2018. This is an Open Access article, distributed under the terms of the Creative Commons Attribution licence (http:// creativecommons.org/licenses/by/4.0/), which permits unrestricted reuse, distribution, and reproduction in any medium, provided the original work is properly cited.

\title{
Nutrition and cancer: prevention and survival
}

\author{
Martin J. Wiseman ${ }^{1,2 *}$ \\ ${ }^{1}$ World Cancer Research Fund International, 22 Bedford Square, London WC1B 3HH, UK \\ ${ }^{2}$ Faculty of Medicine, University Hospital Southampton NHS Trust, University of Southampton, Tremona Road, \\ Southampton SO16 6YD, UK
}

\section{Abstract}

Cancer is increasing worldwide. Patterns of cancer are also changing. The evidence is summarised in the 2018 World Cancer Research Fund/ American Institute for Cancer Research report Diet, Nutrition, Physical Activity and Cancer: a Global Perspective. The plasticity of cancer patterns implicates environmental factors as determinants of cancer, and nutrition influences key cellular and molecular processes that characterise cancer. Epidemiology identifies associations between aspects of diet, nutrition, and physical activity with one or more cancers; there is evidence for plausible mechanisms that imply that these are causal. Some nutritional exposures (alcohol and processed meat) are likely causal factors, but no singular factor protects against cancer (except dietary fibre for colorectal cancer). Cancer protection mainly derives from a systemic metabolic environment that promotes healthy cell replication and tissue integrity. Such a nutritional state reflects avoiding excess adiposity through healthy dietary patterns rich in plant foods (legumes, wholegrains, pulses, vegetables and fruits), with modest meat, fish and dairy, low in alcohol and salt preserved foods, and an active way of life, avoiding sedentary behaviours. Less is known about the impact of nutritional interventions in people with a diagnosis of cancer, but nutrition including adiposity and physical activity predict breast cancer outcome. Promoting healthy ways of life requires public information and education, but alone these do not generate change; a sociopolitical and cultural environment that is conducive to adopting healthy behaviours is needed. Uncertainties in the evidence offer promising directions for future research, but sufficient is known to act as a basis for public policy and clinical practice.

Key words: Diet: Nutrition: Physical activity: Cancer: World Cancer Research Fund/American Institute for Cancer Research expert report

Cancer is the second most important cause of death globally, after CVD. In 2012, there were 14.1 million new cases of cancer, over half of which occurred in less economically developed parts of the world; in 2015 , there were 8.8 million deaths, over two thirds of which occurred in less economically developed areas ${ }^{(1)}$. The WHO estimates that by 2030 , there will be 23.6 million new cases of cancer each year, with the major increase in less economically developed regions of the world ${ }^{(2)}$.

Although all parts of the world are affected by an increasing burden of cancer, the types of cancers that are most common vary considerably from country to country or region to region. For instance, lower-income countries have a greater number of new cases of infection-related cancers (e.g. cervix, liver and stomach). The most commonly diagnosed cancer in men in higher-income countries is prostate, while in less affluent areas, cancers of the oesophagus or stomach are most common ${ }^{(2)}$. In women, breast cancer is most common among both higher- and lower-income countries, but cervical cancer is particularly common in lower-income countries ${ }^{(2)}$.
Lung cancer remains the commonest cancer in men worldwide but is declining in higher-income countries. However in some lower-income countries, reflecting smoking patterns over the last two decades, lung cancer continues to rise, particularly among women ${ }^{(3)}$. Trends in the incidence of various cancers such as prostate, breast and colorectum also vary between higher- and lower-income regions of the world.

One of the most striking features of this global variation in patterns of cancer is that they are not fixed, either in time or place. For instance, when populations migrate to different parts of the world, the cancer patterns among them alters within two generations to conform to that of their host country ${ }^{(4)}$. In addition, the last half century has seen remarkable changes within countries in the incidence of various cancers. For instance, in Japanese men, colorectal cancer was rare until the late 1970s, when incidence began to rise steeply. By the mid 1990s, it had more than doubled but has remained relatively stable since then ${ }^{(5)}$.

The variation in cancer around the world and its relative plasticity are powerful evidence for the importance of environmental factors in determining patterns of cancer worldwide.

Abbreviations: RCT, randomised controlled trial; WCRF, World Cancer Research Fund.

* Corresponding author: Professor M. J. Wiseman, email m.wiseman@wcrf.org 
Furthermore, there is considerable evidence to implicate nutrition as a key factor underlying this phenomenon.

\section{Epistemological considerations}

Most adult cancers are diagnosed after the age of 55 years ${ }^{(6)}$. However, there is evidence that factors operating in early life, marked by height, birth weight or BMI in early life, are predictors of risk of several cancers ${ }^{(7)}$. For colorectal cancer, it is estimated that the process of cancer development extends for decades before diagnosis ${ }^{(8)}$. Establishing the causal factors that might operate over such a long timescale, perhaps with as yet unknown periods of particular sensitivity, poses challenges. In a medical model, convention places randomised trials (or systematic reviews of trials) at the top of a hierarchy of evidence. While a well-designed and executed randomised controlled trial (RCT) offers unique benefits in identifying effects of interventions, free from biases and confounding, conducting one over such a long period is essentially not feasible. Even if such a trial were initiated, attrition would likely differ between control and intervention groups so that over time the special advantages of RCT would be lost.

Furthermore, the exposure of interest (broadly diet and activity) represents a dynamic and complex cluster of exposures, which are variable both within and between people, and over time, and hard to change in a sustained way. Not only it is difficult to conceive of a realistic intervention that might reflect the actual variation in existing dietary or activity patterns, but a difference between study groups over the necessarily prolonged period would be unlikely to be achieved or sustained.

Consequently RCT, with a few exceptions, tend to follow a medical model using dietary supplements rather than actual diet change, over relatively short periods of time, in high-risk subjects, so as to maximise the likelihood of sufficient events for statistical analysis. Even if such a study, well designed and executed, produces clear results, it is often difficult to extrapolate directly from the test intervention to typical populations exposed to usual diet and activity patterns. Thus, while RCT may offer robust answers, they may often be to the wrong question.

For these reasons, much of the evidence relating diet, nutrition and physical activity to the risk of cancer has come from prospective observational data. Such studies are by necessity open to various biases and confounding, which can only be partially overcome by statistical correction. Therefore, deriving causal inference from observed associations is fraught with difficulty. However, for a problem that is significant and potentially preventable, medical and public health practice needs to proceed on the basis of the best evidence, even if that is not perfect (as it never will be, even in the clinical setting with RCT). In analogous circumstances, several authorities have outlined characteristics of observational evidence that help to identify associations that are more, or less, likely to be truly causal. These are exemplified by those outlined by Bradford Hill in the context of occupational disease, but the principles underpinning them can be applied more broadly ${ }^{(9)}$.
Using a structured approach to the epidemiological evidence, and with a cautious interpretation of existing trials, together with consideration of the biological factors that might explain possible causal associations allows judgements on causal and protective factors to be made with reasonable confidence. This represents more a portfolio approach to the evidence rather than a hierarchy, recognising the strengths and weaknesses of different types of study design.

\section{The nature of cancer}

The last decades have seen an explosion of understanding of the molecular events that lead to the development of cancers. The normal control of cell replication, growth and death that helps maintain healthy tissues may become disrupted following genetic or epigenetic alteration in the function of specific genes. Often these genes control cellular functions that were appropriately active during early growth and development, and cancer may be seen as an inappropriate reactivation and distortion of developmental functions in the wrong place at the wrong time. There are a bewildering number of genes and molecular pathways that may be disrupted and lead to cancer $^{(10)}$. Increasingly other components of a tumour beyond the cancer cell are also recognised as important in the development and progression of cancer. However, in spite of the multitude of potentially disrupted molecular pathways, the abnormal behaviour of most cancers can be condensed into eight phenotypic functional domains now known as the Hallmarks of Cancer ${ }^{(10)}$ (Fig. 1).

There is now abundant evidence that nutritional factors, relating to dietary components, body composition in particular body fatness, or levels of physical activity can impact on these characteristics of cells ${ }^{(7)}$. Obesity in particular exemplifies the wide ranging impact that the metabolic disturbances consequent on altered body fatness can have on the cellular and molecular processes that differentiate a cancer cell from a normal cell (Table 1).

Beyond the impact of obesity, several specific dietary components as well as physical activity have been shown to impact on the systemic milieu to render the body more, or less, susceptible to cancer development. Consequently, it is the whole set of nutritional exposures over the life course that is responsible for setting the host susceptibility to exogenous and endogenous carcinogenic exposures (Fig. 2).

In the past, the predominant model for cancer development has been derived from experimental laboratory studies of the carcinogenicity of various external agents. While it is clear that there are environmental carcinogens that have the capacity to overwhelm endogenous protective mechanisms (e.g. metabolism of carcinogens or DNA repair processes), apart from cancers related to smoking or infection, the major burden of cancers in particular in high-income countries, though increasingly in lower- and middle-income countries, is for cancers without major known external causes (e.g. breast and colon). The capacity of the body to cope with the stresses not only from exogenous agents but also from normal oxidative metabolism and cell cycle errors is an important determinant of susceptibility to cancer. Nutritional factors are important in maintaining 
Hallmarks of cancer and two enabling characteristics

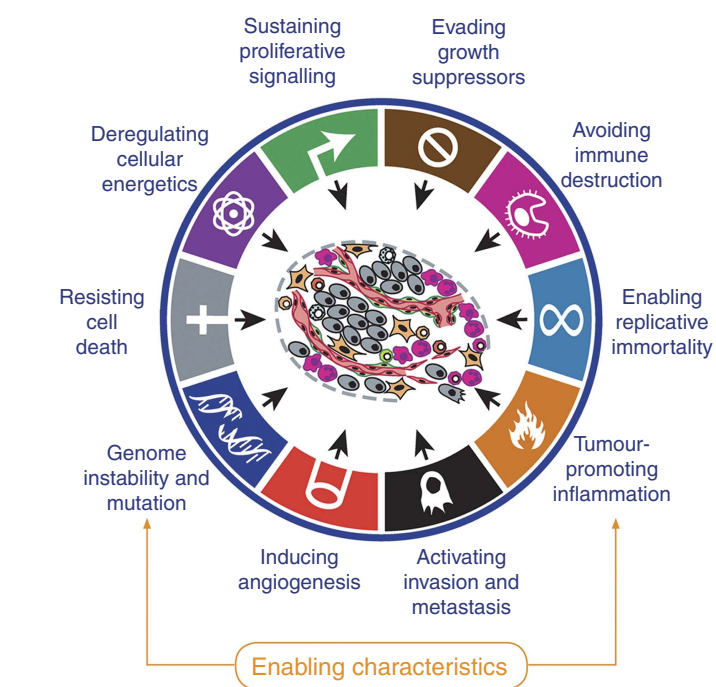

Adapted from: Cell 144, Hanahan D and Weinberg RA, hallmarks of cancer: the next generation, 646-74, Copyright (2011), with permission from Elsevier.

Fig. 1. Hallmarks of cancer. This material has been reproduced from the World Cancer Research Fund/American Institute for Cancer Research. Diet, Nutrition, Physical Activity and Cancer: a Global Perspective. Continuous Update Project Expert Report 2018. Available at dietandcancerreport.org ${ }^{(7)}$. Originally adapted from: Cell 144, Hanahan D and Weinberg RA, Hallmarks of cancer: the next generation, 646-74. Copyright $(2011)^{(10)}$. Despite the multitude of pathways through which genetic damage can lead to the development of cancer, almost all solid tumours can be characterised by a relatively small number of phenotypic functional abnormalities. These eight hallmarks of cancer are facilitated by two enabling characteristics, genome instability and mutation, and tumour-promoting inflammation.

this capacity, while poor nutrition and ageing will tend to reduce it. Therefore, from a nutritional perspective, it is important to consider the overall nutritional state (marked by body composition and the systemic metabolome) than just exposure to specific environmental factors.

External agents, principally smoking, UV radiation and infections, as well as to a lesser extent environmental pollutants, remain important causes of cancer, as do some dietary factors, for example, red and processed meat, or alcohol. However, there is little evidence that single dietary factors are critical in determining protection against cancer, with the exception of wholegrains and dietary fibre in respect of colorectal cancer (which may be a specific local rather than a systemic effect). Rather, it is the contribution of an overall pattern of diet and activity that determines nutritional state (the systemic metabolic milieu and metabolic capacity) marked by body composition including adiposity and the body's consequent capacity to cope with the external or endogenous stresses that promote the development of cancer, which is critical in determining the overall and site-specific risks of cancer.

\section{Nutritional epidemiology of cancer}

The most rigorous analyses of the published literature relating diet, nutrition (including body fatness, usually marked by BMI) and physical activity to cancer risk are those conducted as part of the Continuous Update Project (CUP) of the World Cancer Research Fund (WCRF) International and the American Institute for Cancer Research (AICR). The CUP conducts systematic literature reviews, enters data on a database, performs analyses and displays the results according to a prescribed method developed as part of the 2007 2nd WCRF/AICR Expert Report Food, Nutrition, Physical Activity, and the Prevention of Cancer: a Global Perspective ${ }^{(7)}$. These data are then judged by an independent expert panel who draw conclusions based on predefined criteria to assess the likely causality of observed associations, based on considerations described by Bradford Hill, as discussed above ${ }^{(9)}$. The criteria for judgement include several domains of the overall evidence, including the size and consistency of the effect, the presence of a graded relationship ('dose-response'), the amount and quality of data (e.g. correction for known confounders), the presence of evidence for biological plausibility in humans and correction for potential bias or confounding. The full criteria are found in the WCRF report $^{(7)}$. Based on these judgements, the panel makes recommendations. Recommendations are generally only based on evidence of causality that is regarded as strong (predefined categories of 'convincingly' or 'probably' causal). Other evidence is generally regarded as too limited as a basis for recommendations.

The full systematic literature reviews, as well as a summary of the conclusions and recommendations is published as part of the WCRF/AICR 3rd Expert Report Diet, Nutrition, Physical Activity and Cancer: a Global Perspective ${ }^{(7)}$.

Table 2 presents a summary of those exposures for which there was strong ('convincingly' or 'probably' causal) evidence for an effect on risk of one or more cancers ${ }^{(7)}$.

Although for each of these individual links, the evidence is sufficient to infer with reasonable confidence a truly causal association, they often cluster in dietary and broader lifestyle patterns of behaviour, so that the possibility of residual confounding cannot be entirely excluded. They also may interact physiologically, for example, physical activity and adiposity. Consequently, the greatest confidence can be placed in a pattern of behaviour that optimises as many of these exposures as possible.

While most of the identified exposures can be regarded as modifiable by an individual, this is not true of all. Some, such as As in water or aflatoxin contamination, are beyond the control of most people to influence for themselves. In particular, there are strong signals that factors operating in early life may contribute to later susceptibility to several types of cancer. The striking consistent relation between adultattained heights (which reflects a complex set of influences from before conception to the end of linear growth) is a potent example. It is not yet possible to identify with confidence the whole causal pathway that explains this link, and it is obviously not within any adult's capacity to alter their risk through this means. But understanding the biological pathways that underpin such life course effects offers potential for developing evidence-based guidance on optimal growth trajectories that could be influenced by for instance child feeding practices. This is all the more important to understand 
Table 1. Potential impact of diet, nutrition, physical activity and height in increasing susceptibility to cancer ${ }^{\star}$

\begin{tabular}{|c|c|c|c|}
\hline Exposure & Systemic impact & Cell function & Hallmarks possibly affected \\
\hline \multirow[t]{4}{*}{ Greater body fatness } & Hyperinsulinaemia & mTOR/PI3K/AKT, MAPK & $\begin{array}{l}\text { Reduced apoptosis; increased proliferation; } \\
\text { genome instability }\end{array}$ \\
\hline & Increased oestradiol & MAPK/ERK/PI3K & $\begin{array}{l}\text { Increased proliferation in ER-positive tissues; } \\
\text { genome instability }\end{array}$ \\
\hline & \multirow[t]{2}{*}{ Inflammation } & STAT3/NF- $k$ B & $\begin{array}{l}\text { Reduced apoptosis; increased cell division; altered } \\
\text { macrophage function; genome instability }\end{array}$ \\
\hline & & WNT, P53 & Cellular energetics \\
\hline Greater height & Higher IGF-I & mTOR/PIЗK/AKT, MAPK & Reduced apoptosis; increased proliferation \\
\hline \multirow[t]{4}{*}{ Greater physical activity } & Reduction in insulin & mTOR/PI3K/AKT, MAPK & $\begin{array}{l}\text { Increased apoptosis; reduced proliferation; less } \\
\text { genome instability }\end{array}$ \\
\hline & $\begin{array}{l}\text { Reduction in oestradiol and } \\
\text { testosterone }\end{array}$ & MAPK/ERK/PI3K & $\begin{array}{l}\text { Reduced proliferation in ER-positive tissues; reduced } \\
\text { genome instability }\end{array}$ \\
\hline & \multirow{2}{*}{$\begin{array}{l}\text { Reduced inflammation (long } \\
\text { term); improved immune } \\
\text { function }\end{array}$} & STAT3/NF- $k B$ & $\begin{array}{l}\text { Increased apoptosis; increased cell division; altered } \\
\text { macrophage function; reduced genome instability }\end{array}$ \\
\hline & & WNT, P53 & Cellular energetics \\
\hline $\begin{array}{l}\text { Greater intake of red } \\
\text { and processed meat }\end{array}$ & $\begin{array}{l}\text { Elevated exposure to nitrites; } \\
\text { endogenous } N \text {-nitroso } \\
\text { compound formation }\end{array}$ & $\begin{array}{l}\text { DNA adduct formation } \rightarrow \text { mutations } \\
\text { in p53, KRAS } \\
\text { Oxidative stress, inflammation }\end{array}$ & $\begin{array}{l}\text { Reduced apoptosis; increased proliferation; genomic } \\
\text { instability } \\
\text { Increased inflammation; genomic instability }\end{array}$ \\
\hline $\begin{array}{l}\text { Greater intake of dairy } \\
\text { foods }\end{array}$ & Higher IGF-I & mTOR/PI3K/AKT, MAPK & Reduced apoptosis; increased proliferation \\
\hline \multirow{3}{*}{$\begin{array}{l}\text { Lower vegetables and } \\
\text { fruit intake }\end{array}$} & Folate deficiency & DNA uracil misincorporation & Genome instability \\
\hline & Low dietary fibre intake & Low butyrate & Reduced apoptosis; increased proliferation \\
\hline & $\begin{array}{l}\text { Low levels of carotenoids, } \\
\text { vitamins } A, C, E\end{array}$ & Oxidative stress, inflammation & $\begin{array}{l}\text { Increased inflammation; genomic instability; reduced } \\
\text { apoptosis; increased proliferation }\end{array}$ \\
\hline \multirow[t]{4}{*}{ Greater alcohol intake } & Elevated acetaldehyde & $\begin{array}{l}\text { Oxidative stress, lipid } \\
\text { peroxidation }\end{array}$ & Increased inflammation; genomic instability \\
\hline & Increased oestradiol & MAPK/ERK/PI3K & Increased proliferation in ER-positive tissues \\
\hline & Inflammation & STAT3/NF- $k$ B & $\begin{array}{l}\text { Reduced apoptosis; increased cell division; altered } \\
\text { macrophage function }\end{array}$ \\
\hline & $\begin{array}{l}\text { Folate deficiency; interference } \\
\text { with 1-carbon metabolism }\end{array}$ & DNA uracil misincorporation & Genome instability \\
\hline
\end{tabular}

mTOR, mechanistic/mammalian target of rapamycin; PI3K, phosphoinositide 3-kinase; AKT, also known as protein kinase B; MAPK, mitogen-activated protein kinase; ERK, extracellular signal-regulated kinases; STAT3, signal transducer and activator of transcription 3; WNT, wingless-related integration site; P53, tumour protein p53; KRAS, Kirsten rat sarcoma viral oncogene homologue; IGF-I, insulin-like growth factor 1; ER, oestrogen receptor.

* This material has been reproduced from the World Cancer Research Fund/American Institute for Cancer Research. Diet, Nutrition, Physical Activity and Cancer: a Global Perspective. Continuous Update Project Expert Report 2018. Available at dietandcancerreport.org ${ }^{(7)}$.

as the relationship between adult height and CVD is in the opposite direction $^{(11)}$, probably related to early epigenetic influences.

Although there is a shift in emphasis in the conclusions from the 2018 review compared to that published in 2007, broadly the conclusions are similar. This gives confidence in the robustness of the relationships examined. A systematic review of the link between adherence to these recommendations (as assessed by a scoring system) and cancer incidence, and total or cancer-specific mortality, concluded that there was strong evidence that greater adherence was associated with lower incidence of cancer in general, of cancers of the breast, endometrium and colorectum specifically, and of total mortality, both in men and women ${ }^{(12)}$.

Therefore, the overall body of evidence provides robust evidence that a pattern of diet and activity conforming to the WCRF recommendations helps to reduce risk of several cancers and of total mortality.

\section{After a diagnosis of cancer}

There is mounting evidence that nutritional factors are important predictors of outcome after a cancer diagnosis. Most evidence is in the context of breast cancer, and there are strong associations between obesity and physical inactivity at diagnosis, and worse outcome. There are several possible reasons for this, including inappropriate chemotherapeutic dosing, later diagnosis and worse histopathological features among obese women. However, despite the strong associations (and less strong links with some dietary factors), there is inadequate evidence to conclude that the worse outcome among obese women can be ameliorated by intentional weight loss. The associations may be mediated by several possible confounders that, to date, most observational studies have not taken account of including stage and grade of disease, and therapeutic regimen. A few randomised trials of different dietary and activity approaches are suggestive that survival might be improved by weight management; but because the primary outcomes and tested interventions varied between studies, it is difficult to draw firm conclusions. However, it remains prudent for women to adopt healthy diet and activity patterns, as these improve quality of life and reduce risk factors for cardiometabolic disease. Nevertheless while it is plausible that weight management through healthy diet and activity will improve survival after breast cancer, this cannot currently be concluded with confidence $^{(7)}$.

There is considerably less evidence for other cancers though it is accumulating for prostate and colorectum. What 
Diet, nutrition and physical activity, other environmental exposures and host factors interact to affect the cancer process

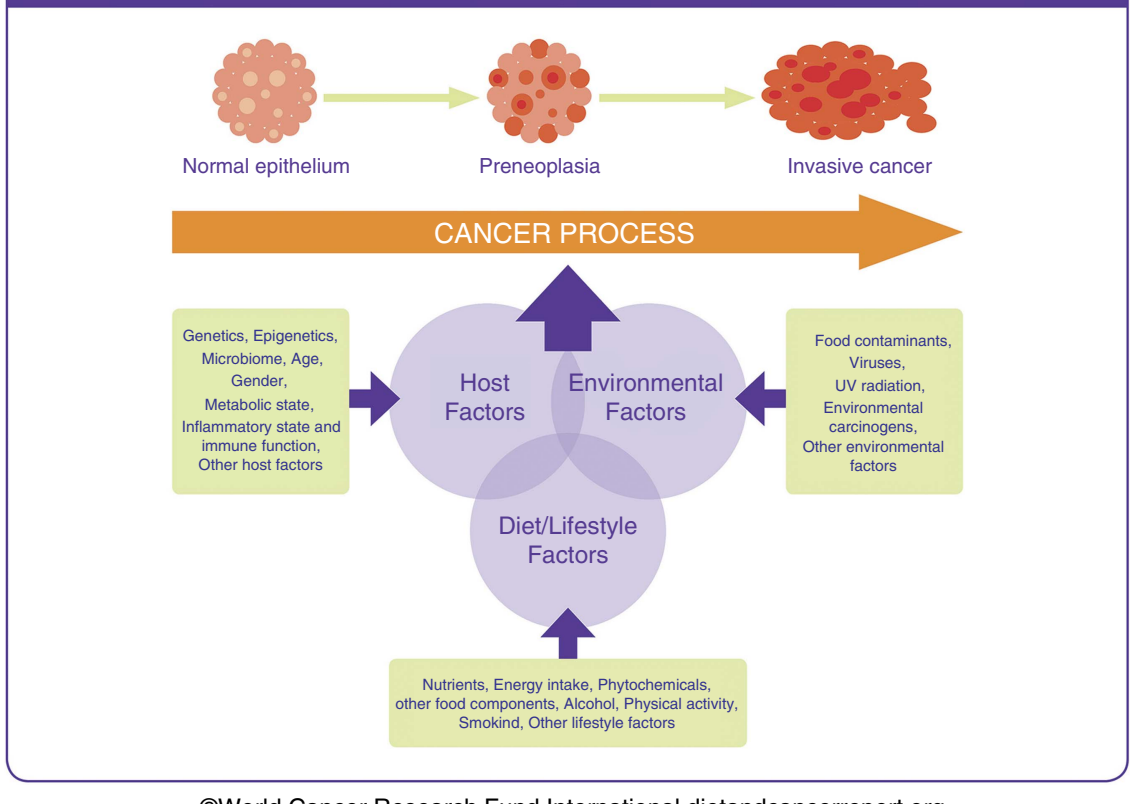

CWorld Cancer Research Fund International dietandcancerreport.org

Fig. 2. The process by which normal cells transform into invasive cancer cells and progress to clinically significant disease typically spans many years. The cancer process is the result of a complex interaction involving diet, nutrition and physical activity, and other lifestyle and environmental factors, with host factors that are related both to inheritance and to prior experience, possibly through epigenetic change. Such host factors influence susceptibility to cancer development, in particular related to the passage of time. This allows both opportunity to accumulate genetic damage, as well as impairment of function, for example, DNA repair processes with ageing. The interaction between the host metabolic state and dietary, nutritional, physical activity and other environmental exposures over the whole life course is critical to protection from or susceptibility to cancer development. This material has been reproduced from the World Cancer Research Fund/American Institute for Cancer Research. Diet, Nutrition, Physical Activity and Cancer: a Global Perspective. Continuous Update Project Expert Report 2018. Available at dietandcancerreport.org ${ }^{(7)}$.

evidence does exist, mostly observational, is also limited by failure to correct for important confounders as for breast cancer.

\section{Cancer in children and adolescence}

The common cancers of adulthood occur mostly after 50 years of age, with an estimated latent period of development of decades. This is the result of the gradual accumulation over time of the necessary genetic and epigenetic modifications to DNA and consequent alteration in the function of genes critical to the control of the cell cycle, cell replication and tissue growth. Clearly, this model cannot apply to the cancers that occur in childhood for which little evidence exists of environmental determinants. People with an inherited genetic predisposition to cancer (e.g. mutations in the $B R C A$ or $A P C$ genes) typically are at higher risk of cancer, which occurs at earlier ages than the sporadic counterparts. It is likely that childhood cancers depend on genetic factors interacting with as yet unknown environmental determinants. However, the successful treatment of many childhood cancers, in particular leukaemias, means that many are surviving into adulthood. Little is known about the impact of nutrition before, at the time of or after diagnosis on the success or otherwise of treatment, on the general wellbeing or on the later health of the child. The challenge of maintaining normal growth and development under the conditions of cancer development and treatment deserves greater attention.

\section{Conclusions}

The variation of the incidence of different cancers between geographical regions, and within regions or countries over time, powerfully implicates environmental factors in determining cancer patterns among populations. Several known factors such as cigarette smoking or infections may explain variation for certain types of cancer but cannot explain the variation in other types of cancer which do not have known major external causes such as colorectal, breast or prostate cancers, which are the leading cancers in higherincome countries. Evidence from many sources, both epidemiological and experimental, implicates nutritional factors (relating to diet and to physical activity, and to the consequent nutritional state including body composition and functional capacity).

Painstaking reviews of the overall body of evidence have identified several singular nutritional exposures (diet, activity or adiposity, as well as markers of early life events) that can be confidently related to risk of one or more cancers. Together these exposures combine to represent a pattern of behaviour that may reduce risk of cancer and of other chronic noncommunicable diseases. The greatest confidence can be placed in the efficacy of a pattern of behaviours that conforms as much as possible to desirable levels of these exposures. 
Table 2. Conclusions from World Cancer Research Fund/American Institute for Cancer Research ${ }^{(7)}$ with strong evidence

\begin{tabular}{|c|c|c|}
\hline Exposure & Direction of effect & Outcomes \\
\hline Greater body fatness & Increases risk & $\begin{array}{l}\text { Cancers of the: } \\
\text { mouth, pharynx and larynx; oesophagus (adenocarcinoma); } \\
\text { stomach (cardia); pancreas; gallbladder; liver; colorectum; } \\
\text { breast (postmenopause); ovary; endometrium; prostate } \\
\text { (advanced); and kidney }\end{array}$ \\
\hline Physical activity & Decreases risk & $\begin{array}{l}\text { Cancers of the: } \\
\text { colon; breast; and } \\
\text { endometrium } \\
\text { Weight gain, overweight and obesity }\end{array}$ \\
\hline Wholegrains & Decreases risk & Colorectal cancer \\
\hline Foods containing dietary fibre & Decreases risk & $\begin{array}{l}\text { Cancers of the colorectum } \\
\text { Weight gain, overweight and obesity }\end{array}$ \\
\hline $\begin{array}{l}\text { 'Fast foods' } \\
\text { 'Western type' diet }\end{array}$ & Increases risk & Weight gain, overweight and obesity \\
\hline Glycaemic load & Increases risk & Endometrial cancer \\
\hline Red meat & Increases risk & Colorectal cancer \\
\hline Processed meat & Increases risk & Colorectal cancer \\
\hline Sugar sweetened drinks & Increases risk & Weight gain, overweight and obesity \\
\hline Alcoholic drinks & Increases risk & $\begin{array}{l}\text { Cancers of the: } \\
\text { mouth, pharynx and larynx; oesophagus (adenocarcinoma); } \\
\text { liver; colorectum; breast; and stomach }\end{array}$ \\
\hline High-dose $\beta$-carotene supplements & Increases risk & Lung cancer (current and former smokers) \\
\hline Breastfeeding (lactation) & Decreases risk & Breast cancer in the mother \\
\hline Breastfeeding (having been breastfed) & Decreases risk & Weight gain, overweight and obesity (children) \\
\hline Greater adult attained height & Increases risk & $\begin{array}{l}\text { Cancers of the: } \\
\text { colorectum; ovary; breast; pancreas; kidney; endometrium; } \\
\text { prostate; and skin (malignant melanoma) }\end{array}$ \\
\hline Greater birth weight & Increases risk & Premenopausal breast cancer \\
\hline As in drinking water & Increases risk & $\begin{array}{l}\text { Cancers of the: } \\
\text { lung; bladder; and skin }\end{array}$ \\
\hline Aflatoxin-contaminated foods & Increases risk & Liver cancer \\
\hline Mate & Increases risk & Oesophageal (squamous cell carcinoma) cancer \\
\hline Foods preserved by salting & Increases risk & Stomach cancer \\
\hline Cantonese-style salted fish & Increases risk & Nasopharyngeal cancer \\
\hline Coffee & Decreases risk & $\begin{array}{l}\text { Cancers of the: } \\
\text { liver; and endometrium }\end{array}$ \\
\hline 'Mediterranean type' dietary pattern & Decreases risk & Weight gain, overweight and obesity \\
\hline Dairy products and $\mathrm{Ca}$ & Decreases risk & Colorectal cancer \\
\hline
\end{tabular}

However, while knowledge of the role of avoiding excess adiposity through healthy diet and activity patterns in reducing risks of chronic non-communicable diseases has been widespread for decades, prevailing behaviours typically do no conform to such a pattern. Knowledge, together with other personal factors such as attitudes and beliefs, can shape people's behaviour but is an insufficient determinant of overall behaviour. The foods people eat and the degree of activity they include in the daily lives on average reflect upstream factors that shape the environment in which people make their choices. The impact of such economic, environmental and social factors is critical in determining the default pattern of behaviour in populations and so creating a social norm which individuals must battle to diverge from (Ref. 2009 WCRF policy report). Attempts to change individual patterns of behaviour significantly from this norm rarely achieve substantial or sustained change. This can only result from changes to the prevailing 'choice environment' that is determined by higher level factors outside the control of individuals but potentially within the power of governments to change or influence. Nutritional public health therefore depends on creating through effective policies an environment that is conducive to people adopting (whether by active choice or by default) healthy patterns of diet and physical activity ${ }^{(7)}$.

There is always more to be learned about the links between diet, nutrition, physical activity and cancer but enough is known to act as a basis for action by individuals and governments.

\section{Acknowledgements}

No financial support was received. M. J. W. is a consultant to WCRF International. M. J. W. declares no conflicts of interest.

\section{References}

1. World Health Organization (2018) Cancer Fact Sheet. http://www.who.int/mediacentre/factsheets/fs297/en/ (accessed August 2018).

2. Forman D \& Bray F (2014) The burden of cancer. In The Cancer Atlas, 2nd ed., pp. 36-37 [A Jemal, P Vineis, F Bray, L Torre and D Forman, editors]. Atlanta, GA: American Cancer Society.

3. Globocan IARC (2018) Lung Cancer. Estimated Incidence, Mortality and Prevalence Worldwide in 2012. http://globocan. iarc.fr/old/FactSheets/cancers/lung-new.asp (accessed August 2018). 
4. Kolonel LN, Hinds MW \& Hankin JH (1980) Cancer patterns among migrant and native-born Japanese in Hawaii in relation to smoking, drinking, and dietary habits. In Genetic and Environmental Factors in Experimental and Human Cancer, pp. 327-340 [HV Gelboin and T no Miya Hi Gan Kenkyū Kikin, editors]. Tokyo: Japan Scientific Society Press.

5. Globocan IARC (2018) GLOBOCAN 2012: Estimated Cancer Incidence, Mortality and Prevalence Worldwide in 2012. Cancer Fact Sheet. http://globocan.iarc.fr/Pages/fact_sheets_ cancer.aspx (accessed August 2018).

6. Cancer Research UK (2018) Cancer incidence by age. http://www.cancerresearchuk.org/health-professional/cancerstatistics/incidence/age (accessed August 2018).

7. World Cancer Research Fund (2018) Diet, nutrition, physical activity and cancer: a global perspective. http://www.wcrf. org/dietandcancerreport (accessed August 2018).
8. Meza R, Jeon J, Renehan AG \& Luebeck EG (2010) Colorectal cancer incidence trends in the United States and the United Kingdom: evidence of right- to left-sided biological gradients with implications for screening. Cancer Res $\mathbf{7 0}$, 5419-5429.

9. Hill $\mathrm{AB}$ (1965) The environment and disease: association or causation? Proc R Soc Med 58, 295-300.

10. Hanahan D \& Weinberg RA (2011) Hallmarks of cancer: the next generation. Cell 144, 646-674.

11. Barker DJ, Gluckman PD, Godfrey KM, et al. (1993) Fetal nutrition and cardiovascular disease in adult life. Lancet $\mathbf{3 4 1}$, 938-941.

12. Kohler LN, Garcia DO, Harris RB, et al. (2016) Adherence to diet and physical activity cancer prevention guidelines and cancer outcomes: a systematic review. Cancer Epidemiol Biomarkers Prev 25, 1-11. 